\title{
Thermal generation of spin current in an antiferromagnet
}

\author{
S. Seki ${ }^{1,2}$, T. Ideue ${ }^{3}$, M. Kubota ${ }^{1,4}$ * Y. Kozuka ${ }^{3}$, R. Takagi ${ }^{1}$, \\ M. Nakamura ${ }^{1}$, Y. Kaneko ${ }^{1}$, M. Kawasaki ${ }^{1,3}$, and Y. Tokura ${ }^{1,3}$ \\ 1 RIKEN Center for Emergent Matter Science (CEMS), Wako 351-0198, Japan \\ 2 PRESTO, Japan Science and Technology Agency (JST), Tokyo 102-8666, Japan \\ 3 Department of Applied Physics and Quantum Phase Electronics Center (QPEC), \\ University of Tokyo, Tokyo 113-8656, Japan and \\ 4 Research and Development Headquarters, ROHM Co., Ltd., Kyoto 615-8585, Japan
}

\begin{abstract}
Longitudinal spin Seebeck effect has been investigated for an uniaxial antiferromagnetic insulator $\mathrm{Cr}_{2} \mathrm{O}_{3}$, characterized by a spin-flop transition under magnetic field along the $c$-axis. We have found that temperature gradient applied normal to $\mathrm{Cr}_{2} \mathrm{O}_{3} / \mathrm{Pt}$ interface induces inverse spin Hall voltage of spin current origin in $\mathrm{Pt}$, whose magnitude turns out to be always proportional to magnetization in $\mathrm{Cr}_{2} \mathrm{O}_{3}$. The observed voltage shows significant enhancement for the lower temperature region, which can be ascribed to the phonon-drag effect on the relevant spin excitations. The above results establish that antiferromagnetic spin waves with high frequency above $100 \mathrm{GHz}$ can be an effective carrier of spin current.
\end{abstract}

PACS numbers: 75.30.Ds, 75.70.-i, 65.40.-b

Spin current, i.e. a flow of spin angular momentum or magnetic moment, has recently attracted revived attention as the potential alternative to charge current with improved energy efficiency [1] 4$]$. Spin-polarized conduction electrons in metallic systems, as well as spin waves in insulating systems, are considered as the two important carriers of spin current [5, 6]. Especially, the latter spin-wave spin current (SWSC) has much longer decay length and can avoid the simultaneous flow of charge current accompanied with Joule heat loss, which are strong advantages for the spintronics applications.

In case of ferro/ferrimagnetic insulators (FMI), SWSC can be generated by various external stimuli such as magnetic resonance 5, 7] or application of temperature gradient $\nabla T[\underline{6}, 8,12$. The latter process is called spin Seebeck effect, and the simultaneous application of $\nabla T$ and magnetic field $H$ to FMI induces SWSC carrying spin angular momentum $\vec{\sigma}(\| \vec{H})$. When paramagnetic metal $(\mathrm{PM})$ is attached to FMI, the spin current $\vec{J}_{s}$ flowing normal to their interface plane is injected into the PM layer through the interfacial spin-exchange interaction $9-12]$. This causes inverse spin Hall effect and associated electric voltage $V_{\mathrm{ISHE}}$ in $\mathrm{PM}$, which is given by

$$
\vec{V}_{\mathrm{ISHE}} \propto L_{V} \theta_{\mathrm{SH}}\left(\overrightarrow{J_{s}} \times \vec{\sigma}\right) .
$$

Here, $\theta_{\mathrm{SH}}$ is the spin-Hall angle of PM, and $L_{V}$ is the gap distance between the electrodes for the voltage measurement. This process can be viewed as a kind of thermoelectric conversion with its efficiency scaling with the film size $L_{V}$, which may offer an unique route for waste heat utilization without requiring a series connection of thermocouples [13.

Previously, the studies of SWSC have mainly focused on a limited number of ferrimagnetic insulators [9] such as rare-earth iron garnet $R_{3} \mathrm{Fe}_{5} \mathrm{O}_{12}$ (including $\mathrm{Y}_{3} \mathrm{Fe}_{5} \mathrm{O}_{12}$ (YIG) [6] and spinel ferrite $M \mathrm{Fe}_{2} \mathrm{O}_{4}$ ( $M: 3 d$ transition metal) 14]. However, the most of magnetic insulators are rather antiferromagnetic [15], and it is a crucial issue whether the spin waves in antiferromagnets can carry spin current or not. Since the dynamics of antiferromagnets are characterized by two or three orders of magnitude higher frequency than those of ferromagnets [16, 17], the antiferromagnetic spin wave can potentially serve as the medium for the ultrafast information processing and communications. Antiferromagnets are also free from stray fields in the ground state, which implies that their dynamics are relatively robust against magnetic perturbations or defects. In general, antiferromagnetic spin wave is described as the propagating precession of two oppositely aligned sub-lattice magnetic moments 16, 17]. Recent theoretical studies have suggested that such local spin oscillations are represented by two degenerated magnon branches carrying opposite sign of spin angular momentum in the limit of $H \rightarrow 0$ [18], where the total spin current will cancel out for the thermal excitation process [19].

In this Letter, we report the experimental observation of spin Seebeck effect for an uniaxial antiferromagnetic insulator $\mathrm{Cr}_{2} \mathrm{O}_{3}$. By applying temperature gradient normal to the $\mathrm{Cr}_{2} \mathrm{O}_{3} / \mathrm{Pt}$ interface, the inverse spin-Hall voltage of spin-current origin has successfully been detected in the Pt layer. The magnitude of thermally induced spin current turns out to be proportional to magnetization in $\mathrm{Cr}_{2} \mathrm{O}_{3}$ even under the $\mathrm{H}$-induced spin-flop transition, proving that antiferromagnetic spin wave characterized by high frequency above a hundred gigahertz can be an effective carrier of spin current.

Bulk single crystals of $\mathrm{Cr}_{2} \mathrm{O}_{3}$ were grown by the laser floating zone method[20]. They are cut into rectangular shape, and polished with diamond slurry and colloidal silica. On the polished surface of $\mathrm{Cr}_{2} \mathrm{O}_{3}$, thin film of $\mathrm{Pt}(10 \mathrm{~nm})$ or $\mathrm{Cu}(20 \mathrm{~nm})$ is deposited as the 

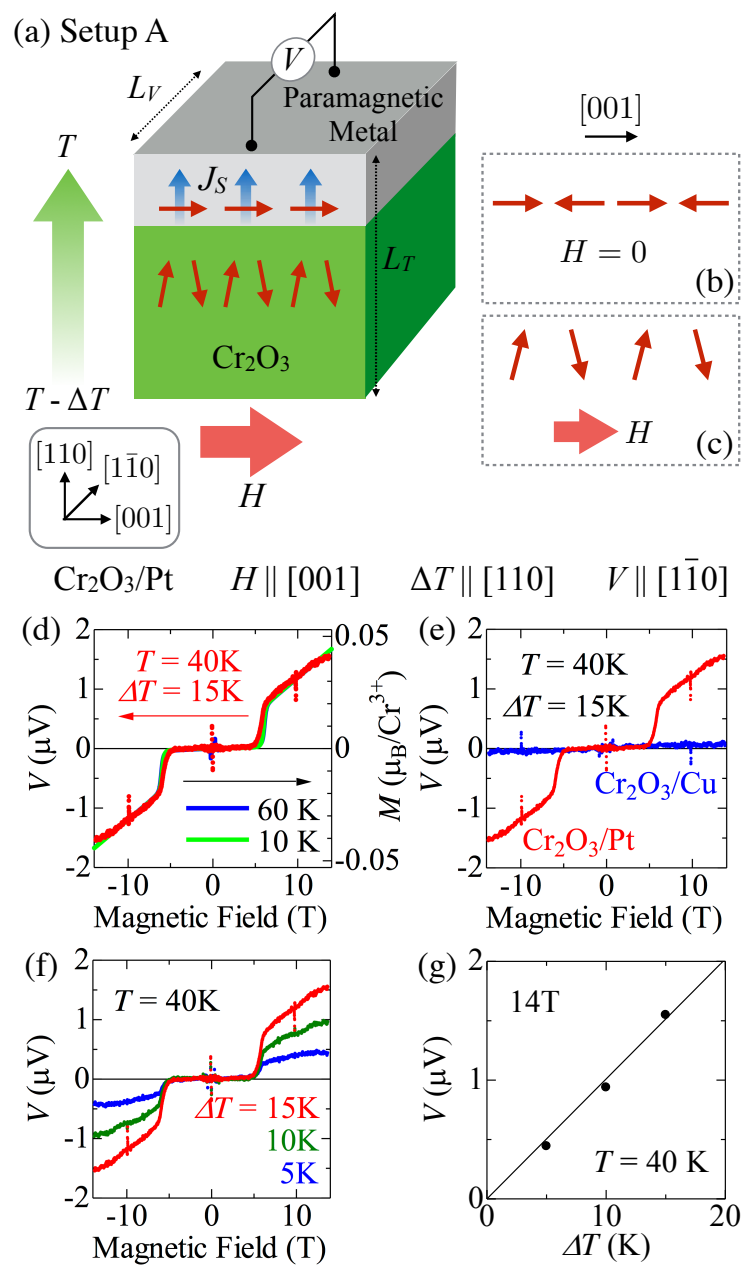

FIG. 1: (color online). (a) Experimental setup for the measurement of longitudinal spin Seebeck effect, with magnetic field $(H)$ applied along the [001] axis of $\mathrm{Cr}_{2} \mathrm{O}_{3}$. Arrows in $\mathrm{Cr}_{2} \mathrm{O}_{3}$ represent the local magnetic moments, and bold blue (thin red) arrows in paramagnetic metal correspond to the propagation direction (carried spin angular momentum) of associated spin current $J_{s}$. $L_{T}$ (thickness of bulk $\mathrm{Cr}_{2} \mathrm{O}_{3}$ along the temperature gradient direction) and $L_{V}$ (distance between the electrodes (black circles) on the metal layer) are $0.5 \mathrm{~mm}$ and $4 \mathrm{~mm}$, respectively. Unless specified, $\mathrm{Pt}$ is employed as the paramagnetic metal. (b) and (c) represent the magnetic structures of $\mathrm{Cr}_{2} \mathrm{O}_{3}$ for the ground state (i.e. $H=0$ ) and the $H$-induced spin flopped state, respectively. (d) $H$-dependence of induced electric voltage $V$ for $\mathrm{Pt}$, and magnetization $M$ for $\mathrm{Cr}_{2} \mathrm{O}_{3}$. The similar voltage profiles are also measured with (e) different paramagnetic metal $(\mathrm{Cu})$ and (f) different magnitudes of temperature gradient $\Delta T$. (g) $\Delta T$ dependence of $\mathrm{Pt}$ voltage at $14 \mathrm{~T}$.

PM layer by radio-frequency sputtering method. To provide the appropriate temperature gradient $\nabla T$, the sample is sandwiched with a pair of $\mathrm{Cu}$ blocks (covered by thin $\mathrm{Al}_{2} \mathrm{O}_{3}$ film to guarantee the electrical insulation but with good thermal contact) under the high vacuum condition less than $10^{-4}$ Torr. One $\mathrm{Cu}$ block serves as the thermal bath with temperature $T-\Delta T$, and another $\mathrm{Cu}$ block is equipped with a resistive heater to keep its temperature $T$. Their temperatures are monitored by cernox thermometers and Lakeshore 335 temperature controller. Here, the temperature gradient is given by $\nabla T=\Delta T / L_{T}$ with $L_{T}$ being the sample thickness along the temperature gradient direction. To evaluate the magnitude of thermally-induced $J_{s}$ through Eq. (11), $H$-dependence of raw electric voltage $V_{\text {raw }}$ are measured in the PM layer with and without $\nabla T$ by nanovoltmeter. After the subtraction of background (i.e. the one with $\Delta T=0)$, the $H$-odd component of induced voltage $V$ is extracted by $V(H, \Delta T)=\left[\left(V_{\text {raw }}(H, \Delta T)-\right.\right.$ $\left.\left.V_{\text {raw }}(H, 0)\right)-\left(V_{\text {raw }}(-H, \Delta T)-V_{\text {raw }}(-H, 0)\right)\right] / 2$. Magnetization $M$ and thermal conductivity $\kappa$ for $\mathrm{Cr}_{2} \mathrm{O}_{3}$ are measured with Physical Properties Measurement System (PPMS, Quantum Design Inc).

The target compound $\mathrm{Cr}_{2} \mathrm{O}_{3}$ has corundum crystal structure with trigonal space group $R \overline{3} c$. The magnetism is dominated by the $\mathrm{Cr}^{3+}$ ion with $S=3 / 2$, and the antiferromagnetic order with local magnetic moments pointing along the [001] axis is stabilized below the Néel temperature $T_{\mathrm{N}} \sim 308 \mathrm{~K}$ (Fig. 1 (b)). Since antiferromagnetically aligned spins prefer to lie normal to $H$, the application of $H \|$ [001] larger than critical field value $H_{c}$ induces spin-flop transition and reorients the magnetic moment direction as shown in Fig. 1 (c) 21, 22].

In the following, we mainly discuss the results for the $\mathrm{Cr}_{2} \mathrm{O}_{3} / \mathrm{Pt}$ sample under the experimental configuration shown in Fig. 1 (a) (i.e. setup A) unless specified. Here, $\mathrm{Pt}$ is deposited on the (110) plane of $\mathrm{Cr}_{2} \mathrm{O}_{3}$ and $\nabla T$ is applied normal to it, which corresponds to the geometry of longitudinal spin Seebeck effect[10, 11]. Magnetic field is applied along the [001] direction of $\mathrm{Cr}_{2} \mathrm{O}_{3}$. To detect the electric voltage of spin current origin following Eq. (11), $V$ component normal to $H$ is measured within the $\mathrm{Pt}$ layer. Figure 1 (d) indicates the magnetic field dependence of $M$ for $\mathrm{Cr}_{2} \mathrm{O}_{3}$, as well as $V$ in the Pt layer at $T=40 \mathrm{~K}$ and $\Delta T=15 \mathrm{~K}$. The application of $H \|[001]$ larger than $H_{c} \sim 6 \mathrm{~T}$ causes a spin-flop transition and magnetization step in the $M-H$ profile, which remains almost $T$-independent below $60 \mathrm{~K}$. Correspondingly, a clear step-like enhancement of $V$ is observed at $H_{c}$. The magnitude of $V$ in $\mathrm{Pt}$ is found to be proportional to $M$ in $\mathrm{Cr}_{2} \mathrm{O}_{3}$, suggesting that the observed voltage originates from thermally-induced spin current mediated by antiferromagnetic spin wave carrying nonzero spin angular momentum $\sigma \propto M$.

To further establish the validity of Eq. (11) in this system, the same voltage measurement is performed for the $\mathrm{Cr}_{2} \mathrm{O}_{3} / \mathrm{Cu}$ sample (Fig. 1(e)). The obtained $V$ in the $\mathrm{Cu}$ layer is negligibly small, consistent with the much smaller spin Hall angle for $\mathrm{Cu}\left(\theta_{\mathrm{SH}} \sim 0.003\right)$ than that for $\mathrm{Pt}$ $\left(\theta_{\mathrm{SH}} \sim 0.1\right) 23$. The measurements are also performed under different magnitudes of $\Delta T$ for the $\mathrm{Cr}_{2} \mathrm{O}_{3} / \mathrm{Pt}$ sample while keeping $T=40 \mathrm{~K}$ (Fig. 1 (f)). Figure 1 (g) 
(a) $\mathrm{Cr}_{2} \mathrm{O}_{3} / \mathrm{Pt} \quad H \|[001]$
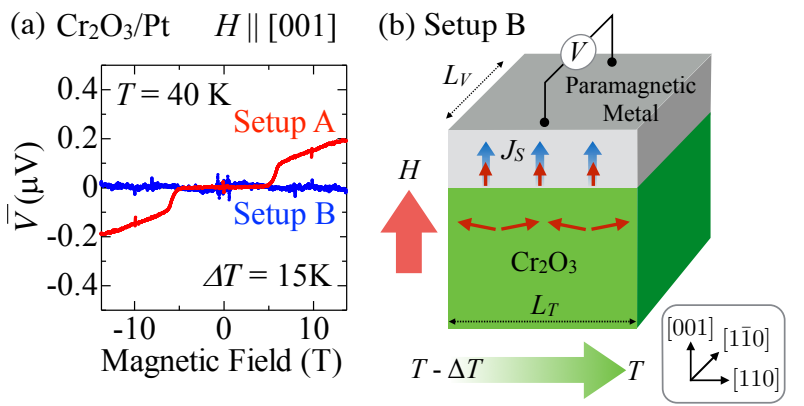

FIG. 2: (color online). (a) Magnetic field dependence of the normalized Pt voltage $\bar{V}\left(=V L_{T} / L_{V}\right)$ for $H$ applied along the [001] axis of $\mathrm{Cr}_{2} \mathrm{O}_{3}$, measured with the setup A (i.e. Fig. 1 (a)) and the setup B as shown in (b). For the latter case, $L_{T}$ and $L_{V}$ are $2 \mathrm{~mm}$ and $4 \mathrm{~mm}$, respectively, and only the anomalous Nernst effect can contribute to the induced voltage.

summarizes the $\Delta T$-dependence of $V$-value at $H=14 \mathrm{~T}$, and $V$ turns out to be proportional to $\Delta T$. Previously, the relationship $J_{s} \propto \nabla T$ has been proposed for several ferromagnetic materials such as YIG[10], and our present results suggest that it also holds for antiferromagnets.

For the YIG/Pt system, the possible contribution of anomalous Nernst effect (ANE) into the $H$-odd voltage component has recently been discussed $[11,24]$. This scenario assumes the proximity ferromagnetism in the $\mathrm{Pt}$ layer (with local magnetization $M_{\mathrm{Pt}}$ ) at the interface with YIG, and the ANE contribution to the voltage is given by

$$
\vec{V}_{\mathrm{ANE}} \propto L_{V}\left(\vec{M}_{\mathrm{Pt}} \times \vec{\nabla} T\right) .
$$

In case of the setup A (Fig. 1 (a)), the observed voltage comprises $V=V_{\mathrm{ISHE}}+V_{\mathrm{ANE}}$ and thus the subtraction of $V_{\mathrm{ANE}}$ is necessary to extract the pure contribution of $V_{\text {ISHE }}$. For this purpose, we employed the different experimental setup as shown in Fig. 2 (b) (i.e. setup B). Here, $\mathrm{Pt}$ is deposited on the (001) plane of $\mathrm{Cr}_{2} \mathrm{O}_{3}$ and $H$ is applied perpendicular to it. $\nabla T$ is along the in-plane [110] direction, and voltage component normal to both $\nabla T$ and $H$ is measured within the Pt layer. In this setup B, $V_{\text {ISHE }}$ becomes zero due to $\vec{J}_{s} \| \vec{\sigma}$ and only $V_{\mathrm{ANE}}$ can contribute to the observed voltage 11]. In Fig. 2 (a), $H$-dependence of $\bar{V}\left(=V L_{T} / L_{V}\right)$, i.e. voltage normalized with sample dimensions, is plotted for the $\mathrm{Cr}_{2} \mathrm{O}_{3} / \mathrm{Pt}$ sample with both setups $\mathrm{A}$ and $\mathrm{B}$. While $H$ induces the spin-flop transition at $6 \mathrm{~T}$ in $\mathrm{Cr}_{2} \mathrm{O}_{3}$ for both configurations, the discernible voltage signal in $\mathrm{Pt}$ is observed only for the setup A. This proves that the contribution of $V_{\text {ISHE }}$ is dominant and $V_{\mathrm{ANE}}$ is negligibly small in the present sample. In case of YIG/Pt with the same $\Delta T$ value, the inverse spin Hall voltage of $\bar{V} \sim$ $3 \mu \mathrm{V}$ has been reported in the saturated ferrimagnetic state at room temperature 11]. The presently observed
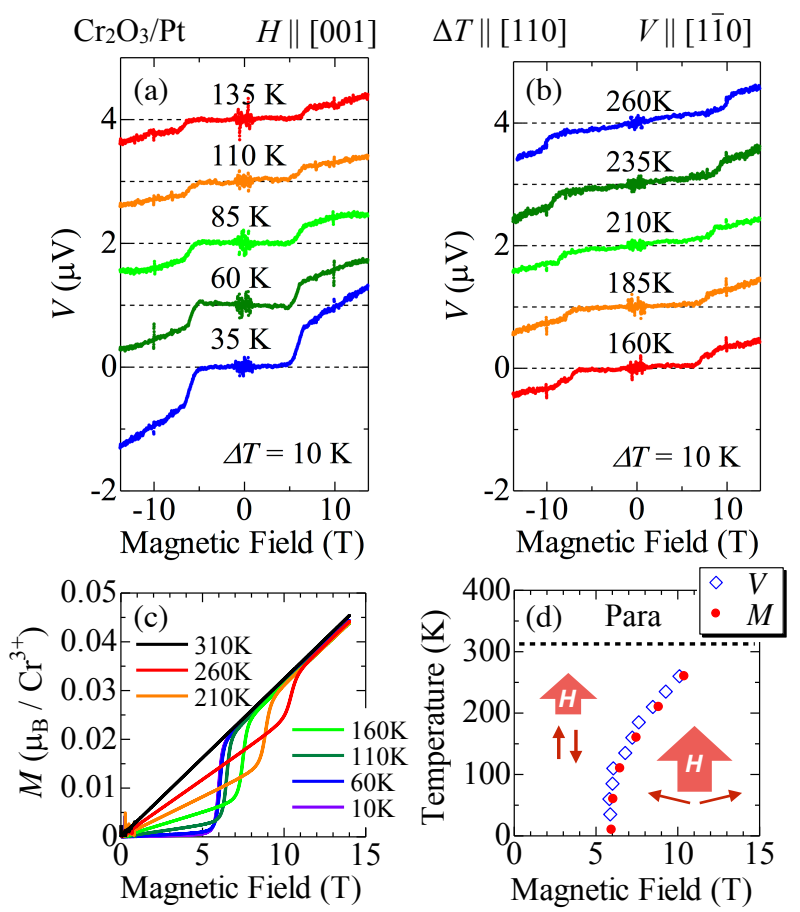

FIG. 3: (color online). (a) and (b) Magnetic field dependence of $\mathrm{Pt}$ voltage measured at various temperatures $T$ for the setup A (i.e. Fig. 1 (a)), with the constant temperature gradient $\Delta T=10 \mathrm{~K}$. (c) The corresponding magnetic field dependence of magnetization for $\mathrm{Cr}_{2} \mathrm{O}_{3}$ with $H \|$ [001]. (d) $H-T$ phase diagram for $\mathrm{Cr}_{2} \mathrm{O}_{3}$ with $H \|$ [001], deduced from anomalies in magnetization profile (red closed circles). Above $T_{\mathrm{N}} \sim 308 \mathrm{~K}, \mathrm{Cr}_{2} \mathrm{O}_{3}$ becomes paramagnetic. The steplike anomalies for Pt voltage observed in (a) and (b) are also plotted as blue open diamonds.

$\bar{V} \sim 0.2 \mu \mathrm{V}$ for $\mathrm{Cr}_{2} \mathrm{O}_{3} / \mathrm{Pt}$ at $14 \mathrm{~T}$ is comparable with the above value, considering the relatively small magnitude of induced $M$ (less than $2 \%$ of saturated magnetization) and the $\bar{V} \propto M$ relationship observed for the thermal excitation process (Fig. 1(d)).

Next, we investigated the temperature dependence of voltage profiles for the $\mathrm{Cr}_{2} \mathrm{O}_{3} / \mathrm{Pt}$ sample with the original setup A. Figures 3 (a) and (b) indicate the $H$-dependence of $V$ in the $\mathrm{Pt}$ layer, measured at various temperatures keeping $\Delta T=10 \mathrm{~K}$. The corresponding $H$-dependence of $M$ for $\mathrm{Cr}_{2} \mathrm{O}_{3}$ obtained at various $T$ is also plotted in Fig. 3 (c). Both $V$ for $\mathrm{Pt}$ and $M$ for $\mathrm{Cr}_{2} \mathrm{O}_{3}$ show a clear step-like anomaly corresponding to the spin-flop transition at $H_{c}$. Based on these measurements, $H-T$ magnetic phase diagram for $\mathrm{Cr}_{2} \mathrm{O}_{3}$ under $H \|$ [001] is summarized in Fig. 3 (d) 22]. While $H_{c}$ becomes larger for higher temperature, the anomalies in $V$ and $M$ always coincide with each other. This confirms that the observed $V$ in $\mathrm{Pt}$ clearly reflects the magnetic nature of underlying $\mathrm{Cr}_{2} \mathrm{O}_{3}$. In Fig. 4 (a), the magnitude of $V$ obtained at $14 \mathrm{~T}$ is plotted as a function of temperature. 

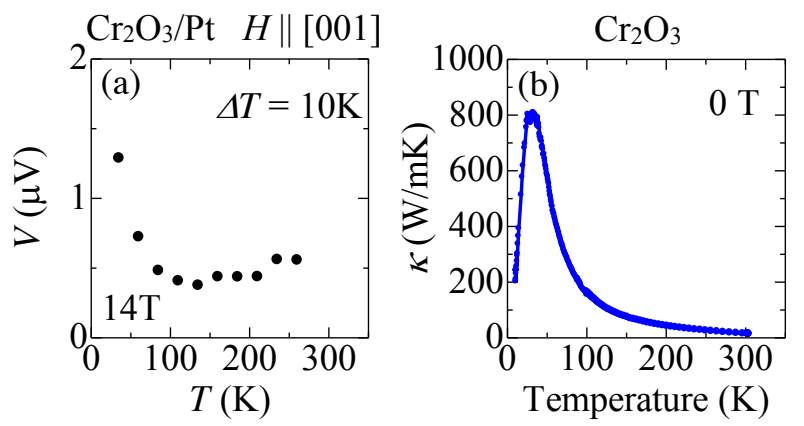

FIG. 4: (color online). (a) Temperature dependence of inverse spin Hall voltage for the $\mathrm{Cr}_{2} \mathrm{O}_{3} / \mathrm{Pt}$ system at $14 \mathrm{~T}$, obtained from the data in Figs. 3 (a) and (b). (b) Temperature dependence of thermal conductivity $\kappa$ along the [110] axis for $\mathrm{Cr}_{2} \mathrm{O}_{3}$, measured under zero magnetic field.

The $V$-value shows clear enhancement for lower $T$, while the corresponding $M$-value at $14 \mathrm{~T}$ remains almost unchanged for whole temperature range as shown in Fig. 3 (c). Similar increase of $V$ for lower $T$ has recently been reported for YIG/Pt, where the relevance of phonon-drag effect has been proposed[9, 25, 26]. This scenario assumes that thermally-induced propagating phonons drag magnons through magnon-phonon interaction, and the prolonged phonon-lifetime $\tau_{\mathrm{ph}}$ in lower- $T$ region enhances the magnitude of $J_{s}$. For insulating materials, the $T$ dependence of $\tau_{\mathrm{ph}}$ can be estimated from thermal conductivity $\kappa$ using the relationship $\kappa=v_{\mathrm{ph}}^{2} C_{\mathrm{ph}} \tau_{\mathrm{ph}} / 3[27,28]$. Here, $v_{\mathrm{ph}}$ represents $T$-independent phonon group velocity, and $C_{\mathrm{ph}}$ does phonon heat capacity whose magnitude monotonically decreases for lower $T$. Figure 3 (d) indicates temperature dependence of $\kappa$ along the [110] axis measured for $\mathrm{Cr}_{2} \mathrm{O}_{3} . \kappa$ shows clear enhancement for lower- $T$ and has maximum around $30 \mathrm{~K}$, which reflects the elongation of $\tau_{\mathrm{ph}}$ for lower $T$ due to the suppression of Umklapp phonon scattering. The present results strongly suggest that the phonon-drag process and the resultant enhancement of $J_{s}$ also take place in antiferromagnets. Note that the $V$-value in $\mathrm{Pt}$ shows slight upturn above $120 \mathrm{~K}$, which may originate from the increase of magnon population.

In general, simple uniaxial antiferromagnets are characterized by two degenerated magnon branches with dispersion relationship $\nu^{+}(k)$ and $\nu^{-}(k)$, which are expected to carry opposite sign of spin angular momentum[18, 19]. This degeneracy is lifted under $H$ applied along the magnetic easy axis, where $\nu^{+}(k)\left(\nu^{-}(k)\right)$ linearly increases (decreases) as a function of $H$. When $\nu^{-}(k)$ reaches zero at $H=H_{c}$, the spin-flopped state characterized by two different magnon branches carrying the same sign of spin angular momentum is stabilized[17]. Since the thermal process excites magnon modes of any wavenumber $k$ and frequency $\nu$ following the Bose distribution function, $\nabla T$ induced generation of nonzero spin current is always jus- tified for antiferromagnets with finite $H$. Note that the typical eigen frequency of antiferromagnetic spin wave ranges from the order of a hundred gigahertz to terahertz, which is much higher than that of ferromagnetic one in the order of gigahertz 17]. In case of $\mathrm{Cr}_{2} \mathrm{O}_{3}$, antiferromagnetic resonance frequency $170 \mathrm{GHz}\left(=\nu^{ \pm}(0)\right)$ has previously been reported for the ground state 30, 31. Considering that $1 \mathrm{~K}$ of thermal fluctuation corresponds to $20.8 \mathrm{GHz}$ of photon frequency, such antiferromagnetic spin waves can be easily excited through the thermal process for the presently employed temperature range. Recent theories predict that each of two degenerated antiferromagnetic magnon branches for $H \rightarrow 0$ is also active for circularly polarized microwave but with opposite handedness 18, 32], and the efficient optical generation of spin current through selective mode excitation under zero magnetic field would be an interesting challenge for antiferromagnets.

In summary, we have experimentally observed longitudinal spin Seebeck effect for an uniaxial antiferromagnetic insulator $\mathrm{Cr}_{2} \mathrm{O}_{3}$. The application of temperature gradient normal to the $\mathrm{Cr}_{2} \mathrm{O}_{3} / \mathrm{Pt}$ interface causes the inverse spin Hall voltage of spin current origin in the $\mathrm{Pt}$ layer, whose magnitude turns out to be proportional to magnetization $M$ in $\mathrm{Cr}_{2} \mathrm{O}_{3}$. The present finding demonstrates that the high-frequency antiferromagnetic spin wave above $100 \mathrm{GHz}$ can be an efficient carrier of spin current, which highlights antiferromagnetic insulators as the promising source of unique spintronic functions.

The authors thank T. Arima, K. S. Takahashi, Y. Iwasa, Y. Kasahara, H. Matsui, Y. Tokunaga, A. Kikkawa, Y. Ohnuma, Y. Shiomi, K. Uchida and E. Saitoh for enlightening discussions and experimental helps. This work was partly supported by the Mitsubishi Foundation, Grants-In-Aid for Scientific Research (Grant No. 26610109, 15H05458) from the MEXT of Japan, and FIRST Program by the Japan Society for the Promotion of Science (JSPS) .

* Present Address: Corporate Technology and Business Development Unit, Murata Manufacturing Co., Ltd., Nagaokakyo, Kyoto 617-8555, Japan

[1] S. Maekawa et al., Spin Current (Oxford University Press, 2012).

[2] S. Murakami, N. Nagaosa, and S. C. Zhang, Science 301, 1348 (2003).

[3] Y. K. Kato et al., Science 306, 1910 (2004).

[4] J. E. Hirsch, Phys. Rev. Lett. 83, 1834 (1999).

[5] Y. Kajiwara et al., Nature 464, 262 (2010).

[6] K. Uchida et al., Nature Mater. 9, 894 (2010).

[7] E. Saitoh, M. Ueda, H. Miyajima and G. Tatara, Appl. Phys. Lett. 88, 182509 (2006).

[8] K. Uchida et al., Nature 455, 778 (2008).

[9] K. Uchida et al., J. Appl. Phys. 111, 103903 (2012).

[10] K. Uchida et al., Appl. Phys. Lett. 97, 172505 (2010). 
[11] T. Kikkawa et al., Phys. Rev. Lett. 110, 067207 (2013).

[12] H. Adachi, K. Uchida, E. Saitoh and S. Maekawa, Rep. Prog. Phys. 76, 036501 (2013).

[13] A. Kirihara et al., Nature Mater. 11, 686 (2012).

[14] K. Uchida et al., Appl. Phys. Lett. 97, 262504 (2010).

[15] M. Imada, A. Fujimori and Y. Tokura, Rev. Mod. Phys. 70, 1039 (1998).

[16] F. Keffer and C. Kittel, Phys. Rev. 85, 329 (1952).

[17] S. Foner, Antiferromagnetic and Ferrimagnetic Resonance, Magnetism 1, 383 (Academic Press, 1963).

[18] R. Cheng, J. Xiao, Q. Niu and A. Brataas, Phys. Rev. Lett. 113, 057601 (2014).

[19] Y. Ohnuma, H. Adachi, E. Saitoh and S. Maekawa, Phys. Rev. B 87, 014423 (2013).

[20] T. Ito et al., J. Cryst. Growth 363, 264 (2013).

[21] M. Fiebig, D. Fröhlich and H. -J. Thiele, Phys. Rev. B 54, R12681 (1996).

[22] R. D. Yacovitch and Y. Shapira, Physica B+C, 86-88,
1126 (1977).

[23] H. L. Wang et al., Phys. Rev. Lett. 112, 197201 (2014).

[24] S. Y. Huang et al., Phys. Rev. Lett. 109, 107204 (2012).

[25] H. Adachi et al., Appl. Phys. Lett. 97, 252506 (2010).

[26] C. M. Jaworski et al., Phys. Rev. Lett. 106, 186601 (2011).

[27] N. W. Ashcroft and N. D. Mermin, Solid State Physics (Holt-Saunders Int., 1976).

[28] For $\mathrm{Cr}_{2} \mathrm{O}_{3}$, the previous thermal conductivity measurement has concluded that its heat transport is dominated by the contribution of phonons 29].

[29] J. M. Jolivet, A. M. de Goer and A. de Combarieu, J. Phys. Collogues 39, C6-990 (1978).

[30] E. S. Dayhoff, Phys. Rev. 107, 84 (1957).

[31] S. Foner, Phys. Rev. 130, 183 (1963).

[32] T. Satoh et al., Phys. Rev. Lett. 105, 077402 (2010). 